\title{
Piecewise linear spine for speed-energy efficiency trade-off in quadruped robots
}

\author{
Mahdi Khoramshahi ${ }^{\mathrm{a}, *}$, Hamed Jalaly Bidgoly ${ }^{\mathrm{a}}$, Soroosh Shafiee ${ }^{\mathrm{a}}$, Ali Asaei $^{\mathrm{a}}$, \\ Auke Jan Ijspeert ${ }^{\mathrm{b}}$, Majid Nili Ahmadabadi ${ }^{\mathrm{a}, *}$ \\ ${ }^{a}$ Cognitive Robotics Laboratory, School of ECE, College of Engineering, University of Tehran, Iran \\ ${ }^{\mathrm{b}}$ Biorobotics Laboratory, Institute of Bioengineering, EPFL, Switzerland
}

\section{H I G H L I G H T S}

- Effects of flexible spine on performance of bounding quadrupeds are studied.

- Superiority of nonlinear spines over linear ones is shown.

- High performance is explained by energy storage-release profile of nonlinear springs.

- Results are justified through a behavioral analogy with biology.

\section{A R T I C L E I N F O}

\section{Article history:}

Received 6 November 2012

Received in revised form

27 July 2013

Accepted 5 August 2013

Available online 22 August 2013

\section{Keywords:}

Robotics

Legged locomotion

Quadruped

Flexible spine

Nonlinear compliance

\begin{abstract}
A B S T R A C T
We compare the effects of linear and piecewise linear compliant spines on locomotion performance of quadruped robots in terms of energy efficiency and locomotion speed through a set of simulations and experiments. We first present a simple locomotion system that behaviorally resembles a bounding quadruped with flexible spine. Then, we show that robots with linear compliant spines have higher locomotion speed and lower cost of transportation in comparison with those with rigid spine. However, in linear case, optimal speed and minimum cost of transportation are attained at very different spine compliance values. Moreover, it is verified that fast and energy efficient locomotion can be achieved together when the spine flexibility is piecewise linear. Furthermore, it is shown that the robot with piecewise linear spine is more robust against changes in the load it carries. Superiority of piecewise linear spines over linear and rigid ones is additionally confirmed by simulating a quadruped robot in Webots and experiments on a crawling two-parts robot with flexible connection.
\end{abstract}

(c) 2013 Elsevier B.V. All rights reserved.

\section{Introduction}

Being stable, fast, energy efficient, adaptable to different terrains, while using simple control and sensory systems, are among the highly desired characteristics of legged robots. A wide range of researches in different domains of robotics and related disciplines have been conducted to realize these characteristics. These researches include the study of animal and human locomotion - from mechanism, material, kinematic, dynamic, and control point of view-, design and construction of diverse stiff locomotion mechanisms, and design and development of different control strategies to control the legged robots. Most of the stiff legged

\footnotetext{
* Corresponding authors.

E-mail addresses: m.khoramshahi@ut.ac.ir (M. Khoramshahi), jalaly.hamed@ut.ac.ir (H. Jalaly Bidgoly), soroosh.shafiee@ut.ac.ir(S. Shafiee), ali.asaei@alumni.ut.ac.ir (A. Asaei), auke.ijspeert@epfl.ch (A.J. Ijspeert), mnili@ut.ac.ir (M. Nili Ahmadabadi).
}

robots have very narrow stability margins, thus a lot of deliberate and accurate computation and control efforts are required to keep the robots stable while walking or running. Energy efficiency of such robots is not satisfactory. Research on control of stiff legged robots made it clear that, unlike manipulators, managing the discontinuity in dynamics is not that easy, while it is easy to have deliberately designed fully or semi-passive biped robots walking at normal speeds [1]. In addition, some researchers showed that, very simple control and oscillatory systems, as in animals, can be used to control semi-passive quadruped robots; see [2-6] as some more recent examples. The passivity of these robots is realized by having springs at the leg joints. The main characteristic of these robots is having a less bumpy dynamic due to having flexibility in their bodies. We call it shaping the robot's dynamics by incorporating simple flexible parts (linear/nonlinear springs) in its body. By shaping the robot's dynamics, stability margins can be broadened. Consequently, the control system can be simplified and adaptation to different terrains can be improved. Especially crafted compliant mechanisms at joints of biped robots are also successfully 


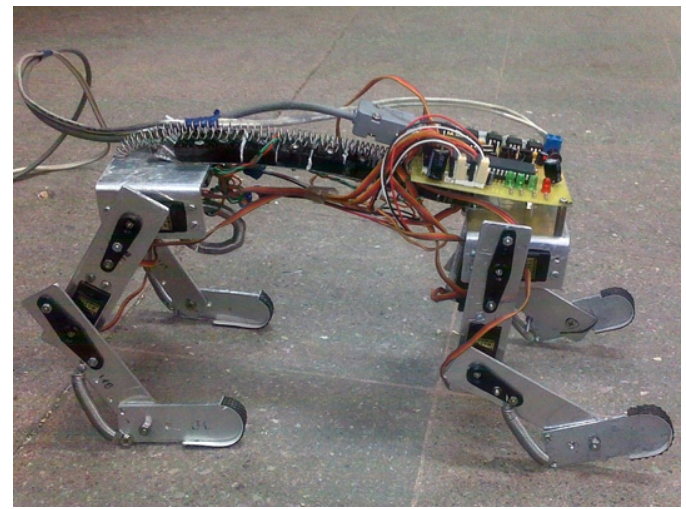

(a) Tiger robot.

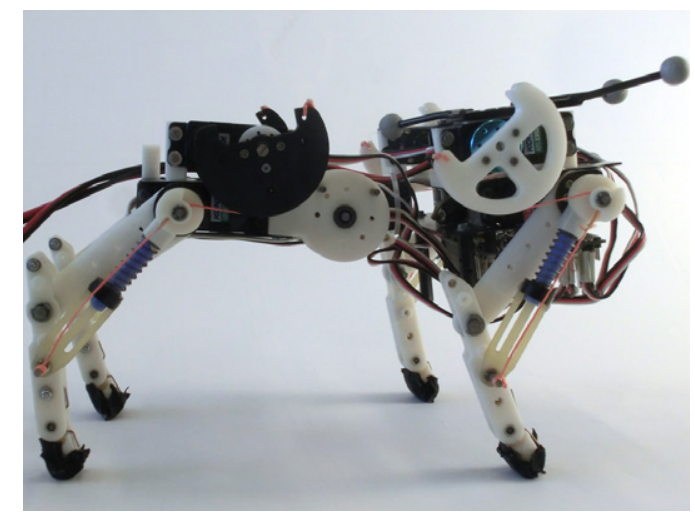

(b) Bobcat robot.

Fig. 1. Tiger, a robot with passive-flexible spine, and Bobcat, a robot with active-flexible spine.

used along with deliberative control systems; see [7]. In addition, it is shown that tuning compliance of active joints through antagonistic setups results in higher efficiency in biped robots $[8,9]$. A mathematical formulation is presented in [10] to find the optimal compliance setting depending on the trajectory and physical properties of system. In [11-13], tendon-driven robots with flexible spine has been preliminary studied. Excluding some recently unveiled robots such as MIT cheetah [14] and Boston Dynamics cheetah [15], most of the existing semi-passive quadruped robots have rigid spines and do not move very fast. It is, however, shown that fast animals, such as cheetahs and tigers, benefit from flexible spines while running [16]. Now the question is how a flexible spine can help a quadruped robot to move fast with a very simple control and sensory system, to have a wide stability margin, and to be energy efficient. In [17], we introduced our Tiger robot, a robot with passive flexible spine (see Fig. 1(a)). It was shown experimentally and by a set of simulations that flexible spine results in more stability, faster movements and less energy consumption compared to the same robot with a stiff spine. Similar results are obtained on a passive quadruped robot with flexible spine [18]. For another comparative research between rigid and flexible spine see [19]. Recently we introduced Bobcat, a small-size quadruped robot with flexible spine [20] (see Fig. 1(b)). Experimental results on Bobcat shows that flexible spine improves velocity and broadens stability margins. In [21], we studied the effects of flexible spine on ground reaction forces. Nevertheless the optimal compliance function of spine is not known. It is shown that compliance in the muscle-tendon units in animal bodies are nonlinear and partially controlled [22]. Therefore, considering the significant role of muscle-tendon behavior in animal's locomotion, one may expect that nonlinear compliant spines have a more positive effect on the locomotion of quadruped robots; compared to the linear spines.

In this research, we study the effect of spine compliance on locomotion performance measures. We look for a proper compliant behavior for spine through a set of simulations and experiments in a simplified locomotion system. Exclusively, we look for a compliant spine that results in a trade-off between the consumed energy and the robot's average velocity. The spine is assumed to be passive and piecewise linear. These assumptions make realization of the spine possible and simple while the overall behavior of spine is still nonlinear. In the rest of this paper, we present a simple model for locomotion in Section 2 and define our objectives in Section 3. The detailed and abstract results of simulations on the model are presented in Sections 4 and 5 respectively. A behavioral analogy between the model and bounding animals is drawn in Section 6 . Experimental and further simulation outcomes are presented in Section 7. In that section, we first test the benefits of using some nonlinear spines in a realization of the model; a crawling robot.

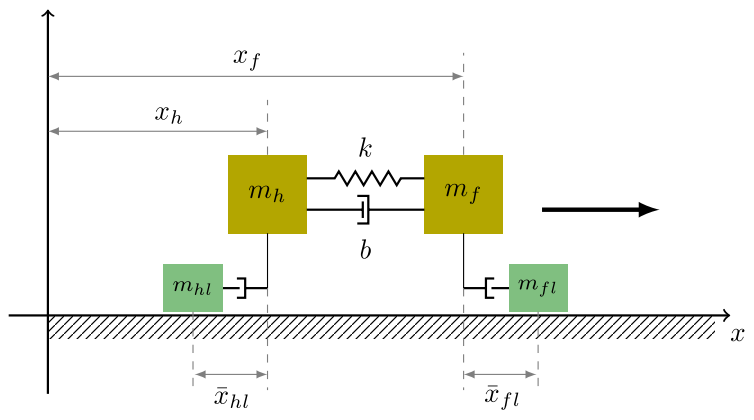

Fig. 2. Model of the locomotion system. Hip and the shoulder masses are connected by a spring and a damper. Each upper mass is connected to the corresponding lower mass by a prismatic actuator. Note that there is only $x$-coordinate.

Then we study the advantages of having nonlinear spine in a Webots model of Ghostdog robot. Discussions and conclusions are presented in Sections 8 and 9 respectively.

\section{Simple model for locomotion}

For locomotion model, we focus on a simplified model of a bounding quadruped; see Fig. 2. Such a model can be found in [23] and the future work section of [24]. Our locomotion system consists of two main parts connected to each other by a spring. The spring models the flexible spine while hind and front parts are analogous to hind and front parts of a quadruped. Each main part comprises two bodies, - namely upper and lower bodies connected to each other by a prismatic actuated joint. The upper bodies model hip and shoulder while lower bodies resemble the role of hind and front legs. In Fig. 2, front(shoulder)/hind(hip) part is denoted by $f / h$, and front/hind leg is denoted by $f l / h l$. In this figure, $m$ and $x$ stand for mass and position respectively.

The relative position of each pair of lower-upper bodies $\bar{x}_{h l}$ and $\bar{x}_{f l}$ are controlled by a hand-tuned PID controller on sinusoidal trajectories with a phase difference. The whole control system can be considered as a simple open-loop CPG-controller (see [25]), thus the amplitude and the frequency of oscillations along with the phase lag determine the locomotion pattern; or so called gait. Another option could be the adaptive Hopf oscillators presented in [26]. Nevertheless, as our focus is on the effect of spine flexibility on fixed gaits, we have opted for simple predetermined sinusoidal trajectories. Any form of locomotion implies an asymmetric interaction between the legs and the ground; see [23]. In legged locomotion, leg-ground interaction can be divided into two asymmetric phases; swing and stance. In our simple model, this asymmetry can be produced via asymmetric friction between lower 
parts and ground. This friction model is not only realistic but also easily realizable. Therefore, we model the leg-ground interaction force with viscous friction where the backward friction is higher than the forward one, i.e.:

$F_{\text {fric }}(v)= \begin{cases}-f_{\text {forward }} & v_{\text {leg }}>0 \\ 0 & v_{\text {leg }}=0 \\ +f_{\text {backward }} & v_{\text {leg }}<0\end{cases}$

where $F_{\text {fric }}$ is the friction force, $v_{\text {leg }}$ is the leg velocity with respect to the ground and $f_{\text {forward }}<f_{\text {backward }}$. Having higher backward friction is consistent with stance phase in quadruped locomotion and $f_{\text {for ward }}=0$ conceptually models leg lift up and forward swing. Dynamic equations for the legs are:

$$
\left\{\begin{array}{l}
F_{\text {pid }}\left(\bar{x}_{h l}, \bar{x}_{h l}^{\text {desired }}\right)-F_{f r i c}\left(\dot{\bar{x}}_{h l}+\dot{x}_{h}\right)=m_{h l}\left(\ddot{\bar{x}}_{h l}+\ddot{x}_{h}\right) \\
F_{\text {pid }}\left(\bar{x}_{f l}, \bar{x}_{f l}^{\text {desired }}\right)-F_{f r i c}\left(\dot{\bar{x}}_{f l}+\dot{x}_{f}\right)=m_{f l}\left(\ddot{\bar{x}}_{f l}+\ddot{x}_{f}\right)
\end{array}\right.
$$

where $F_{\text {pid }}$ is the force generated by the PID controller. For the upper bodies we have:

$$
\left\{\begin{array}{l}
-F_{\text {pid }}\left(\bar{x}_{h l}, \bar{x}_{h l}^{\text {desired }}\right)+k\left(x_{f}-x_{h}\right)+b\left(\dot{x}_{f}-\dot{x}_{h}\right)=m_{h} \ddot{x}_{h} \\
-F_{p i d}\left(\bar{x}_{f l}, \bar{x}_{f l}^{\text {desired }}\right)-k\left(x_{f}-x_{h}\right)-b\left(\dot{x}_{f}-\dot{x}_{h}\right)=m_{f} \ddot{x}_{f}
\end{array}\right.
$$

where $k$ and $b$ are the coefficients of spring and damper located between the two upper bodies; namely the spine properties. Ultimately, our system is parameterized by CPGs parameters (frequency, amplitude, phase lag), environment parameters (forward/backward friction constants) and body parameters (mass of upper/lower parts and spine compliance). In the following simulations, mass of each upper body/leg is $3 \mathrm{~kg} / 1 \mathrm{~kg}$, the damping coefficient is set to $0.1 \mathrm{~N} \mathrm{sm}^{-1}$, and forward $\left(f_{\text {forward }}\right)$ and backward friction $\left(f_{\text {backward }}\right)$ coefficients are set to $0.2 \mathrm{~N}$ and $0.4 \mathrm{~N}$ respectively. For PID controller, we have $K_{p}=20, K_{i}=0.1$, and $K_{d}=5$. Extensive simulations showed that the optimal phase difference for all cases is $180^{\circ}$. However, this phase lag is in line with bounding gait in animals where movement of spine is salient. Therefore, all of the reported results are done using this phase difference value.

\section{Problem statement}

Finding the best and easily realizable compliant spine that improves both energy efficiency and speed is the main goal of this paper. As a measure for the energy efficiency, we use cost of transportation (COT) which is widely used in the literature (see [27-29]). This dimensionless index can be calculated as follows:

$\operatorname{COT}=\frac{E_{\text {cons }}}{m g d}$

where $m$ is the total mass, $g$ is the gravitational constant, $d$ is the traveled distance, and $E_{\text {cons }}$ is the consumed energy defined as:

$E_{\text {cons }}=\int_{0}^{t}\left[\left|F_{p i d}\left(\bar{x}_{h l}, \bar{x}_{h l}^{\text {desired }}\right) \cdot \dot{\bar{x}}_{h l}\right|+\left|F_{\text {pid }}\left(\bar{x}_{f l}, \bar{x}_{f l}^{\text {desired }}\right) \cdot \dot{\bar{x}}_{f l}\right|\right] d t$.

It means that no energy is retrieved while braking by the actuators; the same is true in quadrupeds and most of the existing robots. So the goal is to minimize the negative work by choosing a proper compliance function [30]. In the experiments and in the simulations, we give a fixed amount of energy (200 J in the simulations) to the system. Using the travel time and distance, we can easily calculate COT and robot's average velocity. To have a better resolution, we present our results in $1 /$ COT instead of COT. Therefore, our goal is to maximize both indexes, $1 /$ COT and $\bar{v}$.

Velocity-COT trade-off

Velocity-COT trade-off is well-known in legged locomotion, and it explains the existence of multiple gaits in animals; see [31]. Animals exhibit different gaits in order to be energy efficient in different regions of velocity. Only considering velocity in our work will lead to fast gaits with huge amount of energy consumption, and only considering COT will result in efficient but slow gaits. However in reality, sources of energy are limited and energy efficiency plays an important role as well as velocity, and it will be shown that designing the proper compliance can improve this trade-off.

\section{Simulation results on the simple model}

Our goal is to study the effect of having compliance in the spine, so the gait needs to be fixed; namely the frequency $(f)$ and the amplitude $(A)$ of $\bar{x}_{h l}$ and $\bar{x}_{f l}$. A rough investigation over different values of linear spine spring constant $(k), f$ and $A$ is done to set these two parameters. Fig. 3(a) and (b) shows justifications for choosing $A=0.8 \mathrm{~m}$ and $f=1 \mathrm{rad} / \mathrm{s}$ in order to achieve fast locomotion and acceptable COT over a range of linear spines. However, based on our extensive simulations, the conclusions we make in this paper are valid for wide and reasonable range of gaits. Fig. 3(a) shows the effect of oscillation frequency on the speed and COT for different spring constants when $A=0.8 \mathrm{~m}$, while Fig. 3(b) shows the effect of amplitude when $f=1 \mathrm{rad} / \mathrm{s}$. In these figures, $k=0 \mathrm{Nm}^{-1}$ means that the upper parts are not connected.

In Fig. 3(a) and (b), we can see that up to a point (2 rad/s), speed and COT work against each other. Our choice of frequency and amplitude is to satisfy this trade-off. It can be seen from these figures that $1 /$ COT decreases asymptotically. COT is proportional to $E_{c o n}$. For oscillatory point-mass object (legs in this model), $E_{c o n}$ can be calculated as follows:

$$
\begin{aligned}
& \left\{\begin{array}{l}
x(t)=A \sin (\omega t) \\
\dot{x}(t)=A \omega \cos (\omega t) \\
\ddot{x}(t)=-A \omega^{2} \sin (\omega t) \\
F(t)=m \ddot{x}(t)
\end{array}\right. \\
& E_{\text {cons }}=\int_{0}^{2 \pi / \omega}|F(t) \dot{x}(t)| d t=2 m A^{2} \omega^{2} .
\end{aligned}
$$

It can be concluded that $1 / C O T$ is proportional to $A^{-2}$ and $f^{-2}$, and this explains the asymptotic behavior of $1 / C O T$ graphs in Fig. 3(a) and (b). Average velocity is more related to asymmetric friction. In order to transform oscillatory motion (of legs) to only forward motion (of body), average forces generated by legs should fall in $\left[F_{\text {forward }}, F_{\text {backward }}\right]$. Forces less than $F_{\text {forward }}$ are not enough to push the body forward. Forces more than $F_{\text {backward }}$ are too high that can push the robot backward and cause large oscillatory motion in body. Although this motion, in average, moves the robot forward, but it is slow. Since the average force created by legs are proportional to $A^{2}$ and $\omega^{2}$, for a given frequency, we expect to see a bell shape behavior in velocity graphs in Fig. 3(a) and (b).

\subsection{Linear spring}

Now that every parameter but the spring constant is fixed, we can study the effect of compliance; Fig. 3(c) shows the average speed and the energy efficiency over a wide range of linear spring constants. Important points in these graphs are summarized in Table 1.

As Table 1 shows, the spring constants for the best $1 / C O T$ and the fastest cases are very different; namely $54 \mathrm{Nm}^{-1}$ and $2.2 \mathrm{Nm}^{-1}$ respectively. A comparison with the rigid spine shows that both the energy efficiency $(1 / C O T)$ and the velocity can be improved by using a linear flexible spine. Nevertheless, the improvement in locomotion speed $(87.5 \%)$ is much higher than the enhancement in energy efficiency (8.9\%). Additionally, in this system, as in a running cheetah, the speed of locomotion is highly depended on the spine flexibility. Having tested linear springs, we should check if better solution can be attained using a nonlinear spring. 

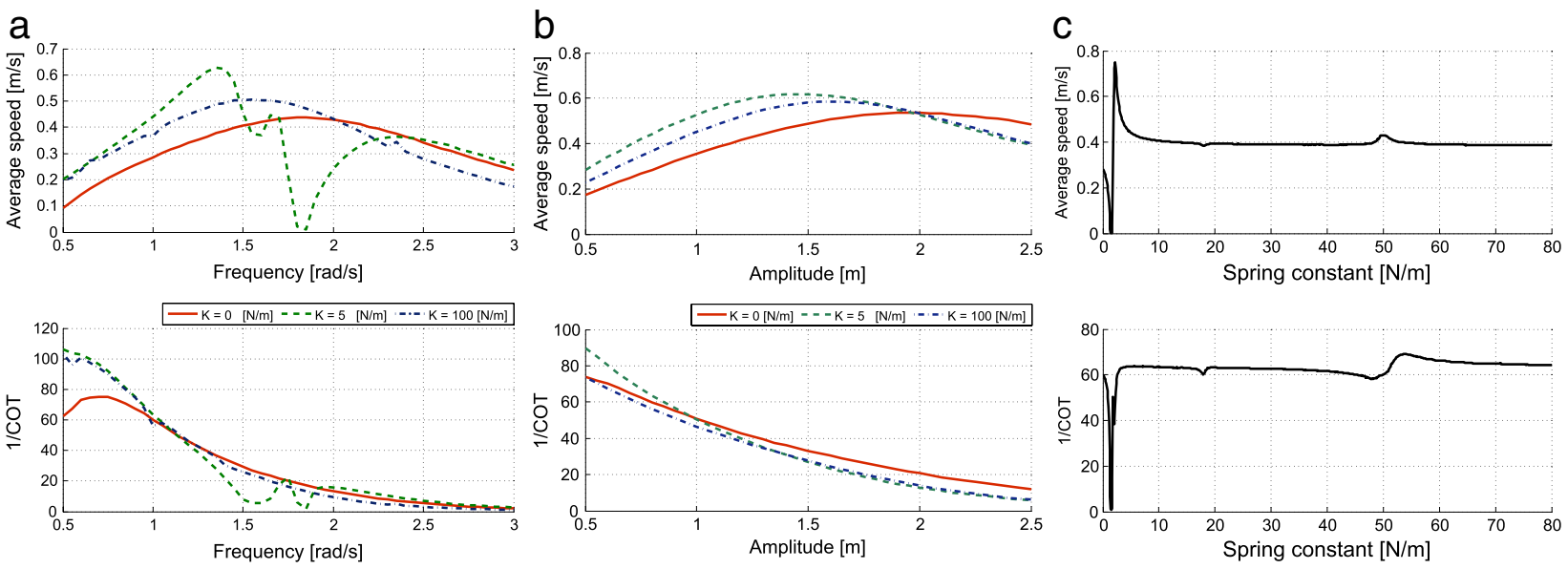

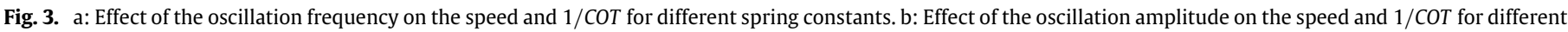
spring constants. c: The effect of spine stiffness on the energy efficiency and the locomotion velocity for the nominal gait.

a

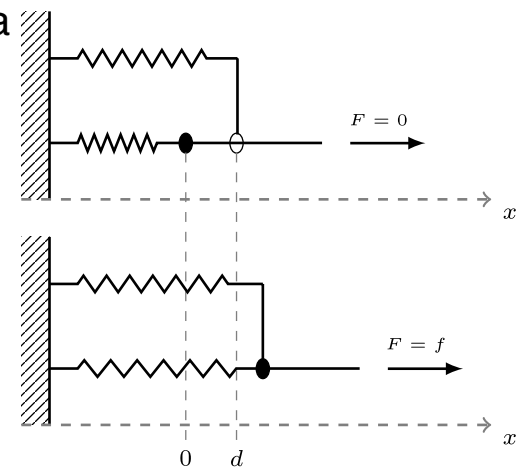

b

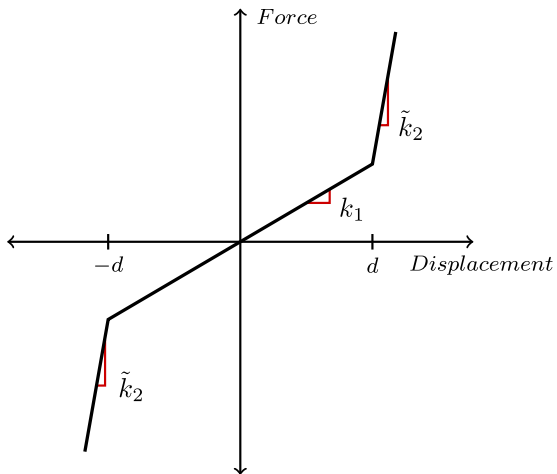

Fig. 4. a: Realization of a nonlinear (piecewise linear) spring. b: Symmetric nonlinear (piecewise linear) spring with three parameters, $k 1, k 2$ and $d$.

Table 1

Result of linear and nonlinear spine.

\begin{tabular}{|c|c|c|c|c|c|c|c|}
\hline \multirow[t]{2}{*}{ Case } & \multicolumn{3}{|l|}{ Linear } & \multicolumn{4}{|l|}{ Nonlinear } \\
\hline & $K\left(\mathrm{Nm}^{-1}\right)$ & $1 / \mathrm{COT}$ & $\bar{v}\left(\mathrm{~ms}^{-1}\right)$ & $k_{1}\left(\mathrm{Nm}^{-1}\right)$ & $d(\mathrm{~m})$ & $1 / \mathrm{COT}$ & $\bar{v}$ \\
\hline Best COT & 54 & 69.03 & 0.40 & 0 & 0.55 & 77.78 & $0.54 \mathrm{~m}^{-1}$ \\
\hline Fastest locomotion & 2.2 & 46.38 & 0.75 & 2.2 & 3.00 & 47.13 & $0.75 \mathrm{~ms}^{-1}$ \\
\hline
\end{tabular}

\subsection{Piecewise linear spring}

In this section, we check if making the spine's stiffness nonlinear improves the robot's locomotion performance. Having more degrees of freedom in compliance could be an advantage for performance optimization. To simplify the search, we look for the best piecewise linear spring. The second rationale behind this choice is that piecewise linear springs can be made easily; see Fig. 4(a) as one of the possible ways to construct such springs and [10] for a variety of designs. A synthesis method for custom compliance profile is presented in [32]. In this figure, we have two linear springs in parallel. The first spring $\left(k_{1}\right)$ is engaged for all displacements while the second one $\left(k_{2}\right)$ gets connected for deflections larger than a specified value; i.e. $x>d$. It results in the following stiffness profile.

$K(x)= \begin{cases}k 1 & x<=d \\ \overbrace{k 1+k 2}^{\tilde{k}_{2}} & x>d .\end{cases}$

By choosing a piecewise linear spring, search for the best nonlinear spring is reduced to a search for a limited number of stiffness values and the displacements where each spring gets engaged. By assuming symmetry for spring profile, the number of parameters drops dramatically. Our choice of the nonlinear spring is illustrated in Fig. 4(b). It is also interesting to know that $K(x) \stackrel{d \rightarrow \infty}{\longrightarrow} k_{1}$ and $K(x) \stackrel{d \rightarrow 0}{\longrightarrow} \tilde{k}_{2}$.

Being inspired by the compliance behavior of muscle-tendon, we set a relatively high stiffness $\left(\tilde{k}_{2}=20 \mathrm{Nm}^{-1}\right)$ for the second spring. Using a stiff spring is not an unusual issue. In chapter 5 of [10], it is reported that for some instants, infinite stiffness is the best choice. The search space is now two dimensional; namely the spring constants and the engagement location for the hard/stiff spring. Grid search for the proper nonlinear spring is illustrated in Fig. 5(a) and (b); important cases are summarized in Table 1.

Fig. 5(a) shows that the best energy efficiency $(1 / C O T)$ is achieved by using a fully compliant first spring $\left(k_{1}=0 \mathrm{Nm}^{-1}\right)$ when the stiff spring $\left(\tilde{k}_{2}=20 \mathrm{Nm}^{-1}\right)$ is engaged at $0.55 \mathrm{~m}$; see Table 1. Furthermore, the good point about the energy efficiency curve is being flat around the optimal point. It means robustness in terms of energy efficiency against the change in the compliance of the spring and its engagement location. First row of Table 1 shows $11.16 \%$ improvement in $1 /$ COT by using piecewise linear spring. Fig. 5(b) shows that the best spring for fast locomotion is relatively 
a

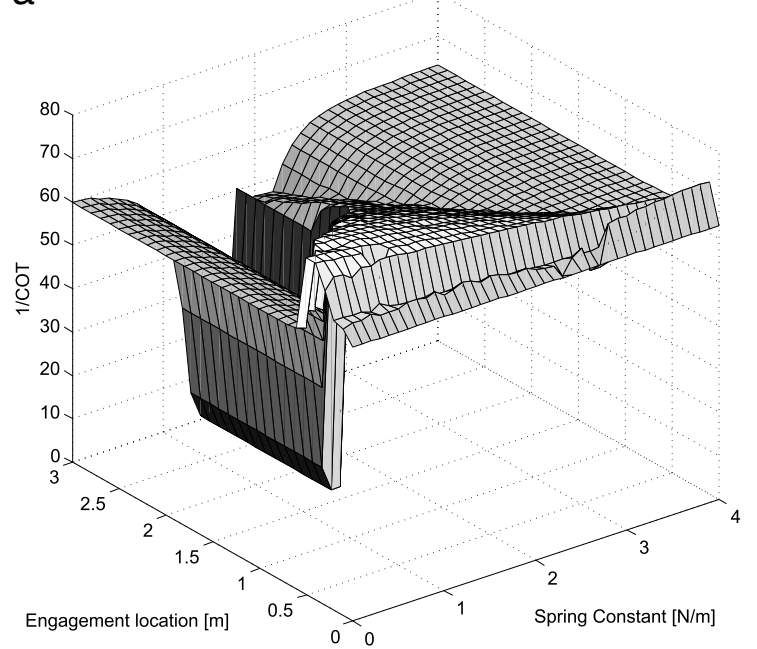

b

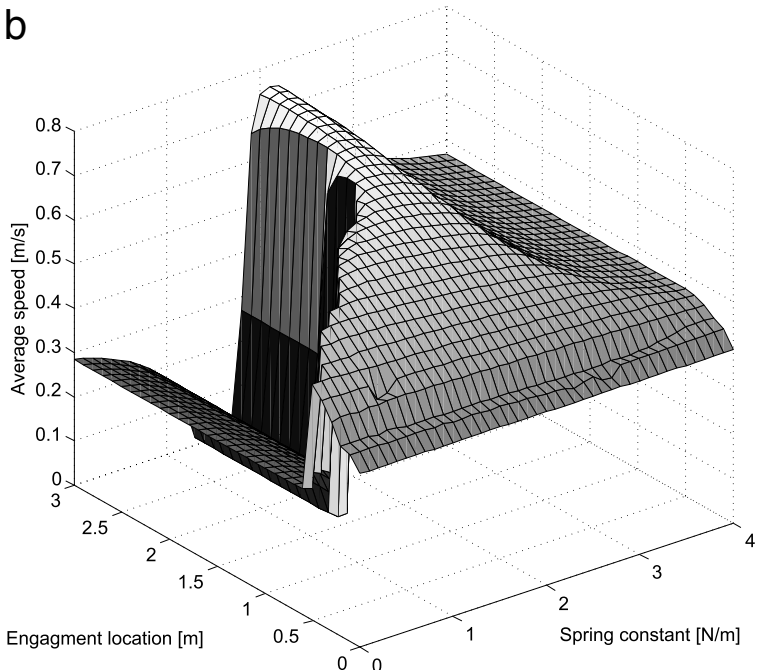

Fig. 5. a: Effect of spring constant $\left(k_{1}\right)$ and engagement location of second spring $(d)$ on energy efficiency $(1 / C O T)$. b: Effect of spring constant $\left(k_{1}\right)$ and engagement location of second spring $(d)$ on average velocity $(\bar{v})$.
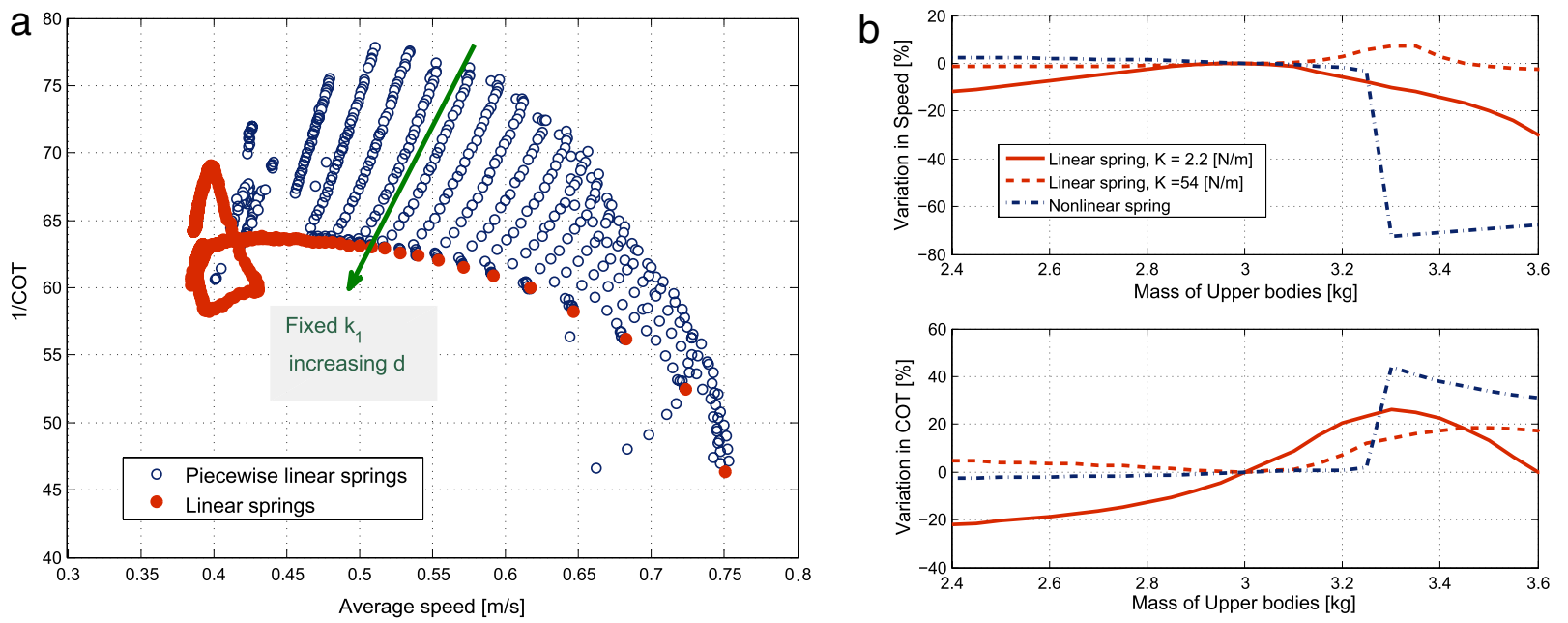

Fig. 6. a: Representation of the linear and piecewise linear spine performances in Pareto fashion. b: Effects of the mass changes on the locomotion performance of the robot with linear and the piecewise linear (nonlinear) spines.

soft and the engagement location of the second spring is quite far. Far enough to resemble the linear spring for fastest locomotion; check $K$ and $k_{1}$ in the second row of Table 1. Finally, it can be seen that the energy efficiency and velocity cannot be simultaneously optimized using piecewise linear springs.

To check if increasing the number of springs enhances the performance, a GA optimization for symmetric 5 pieces of springs was performed and the improvement was very small. Therefore, hereafter we focus on our symmetric 3 pieces spring system.

\section{Comparison}

\subsection{Energy efficiency and locomotion speed}

The simulation results showed that there is no single compliance, neither in the linear nor in the piecewise linear cases, which could maximize the energy efficiency and velocity simultaneously. Therefore, the linear and the piecewise linear spines are compared through contrasting Pareto-optimal points [33] of the two cases; see Fig. 6(a). Interesting point about this figure is that the data of each nonlinear spine is above and to the right of the corresponding linear spine's data. The corresponding linear spine is obtained by increasing $d$ such that the second spring is not activated at all. It means that for each Pareto point of the linear spine, there is a Pareto point of the piecewise linear spine that results in higher locomotion speed and better energy efficiency. In conclusion, using piecewise linear spine can lead to both faster and more energy efficient locomotion in comparison to employing the linear spine.

Another interesting observation is that for every fixed value of $k_{1}$, decreasing the engagement location of the second spring $(d)-$ from the point that the second spring becomes effective down to a limit - results in both higher energy efficiency and faster locomotion; see Fig. 6(a). However, improvement in the energy efficiency is more significant. Interestingly, in the sequel, we report the same observation for the quadruped robot in Webots simulation.

\subsection{Robustness against mass changes}

In this section, the effect of change in the upper body masses on the locomotion performance is investigated. Changing mass of upper bodies is interesting because it resembles load carrying situation. Here we change the masses of hip and shoulder from $2.4 \mathrm{~kg}$ to $3.6 \mathrm{~kg}$ and evaluate the energy efficiency and the locomotion velocity in three different cases: 
a
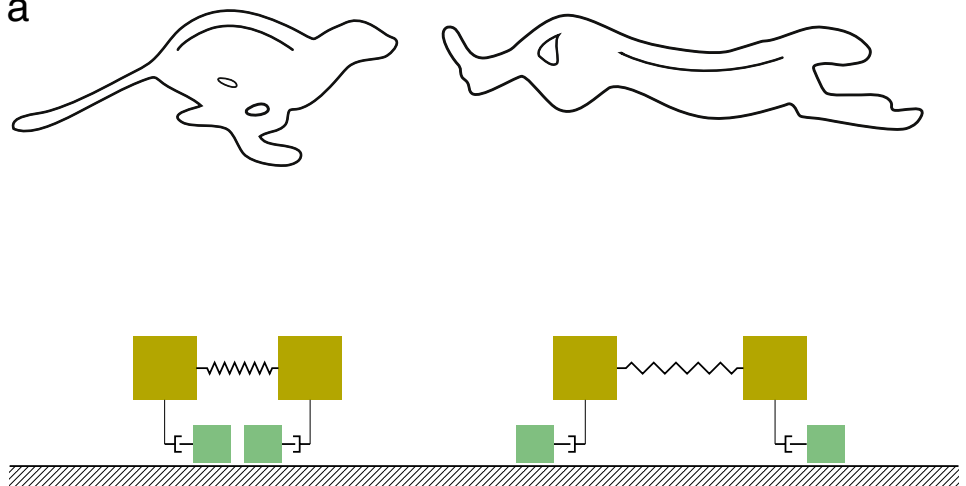

b

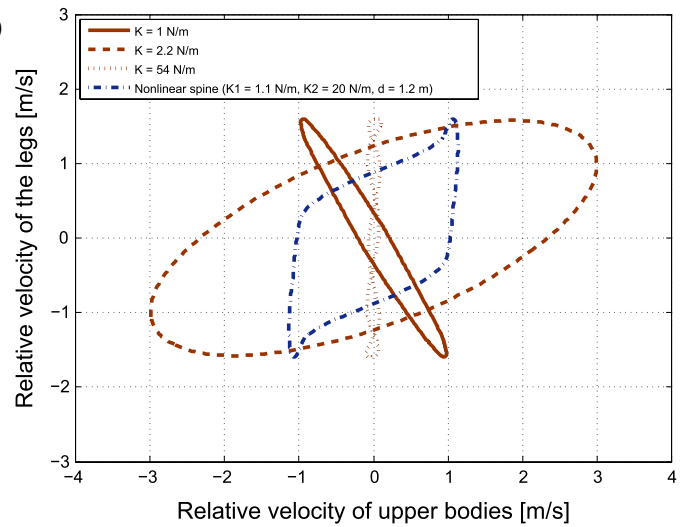

Fig. 7. a: Two state of cheetah and simple model; cheetah stick figures are drawn based on [34] b: Effect of the oscillation amplitude on the speed and 1/COT for different spring constants.

Case1: linear spine with the fastest locomotion speed

$$
\left(k_{1}=2.2 \mathrm{Nm}^{-1}\right) \rightarrow\left(\bar{v}=0.75 \mathrm{~ms}^{-1}, 1 / \mathrm{COT}=46.38\right) .
$$

Case2: linear spine with highest energy efficiency

$$
\left(k_{1}=54 \mathrm{Nm}^{-1}\right) \rightarrow\left(\bar{v}=0.40 \mathrm{~ms}^{-1}, 1 / \mathrm{COT}=69.03\right) .
$$

Case3: nonlinear spine for the fastest locomotion at $90 \%$ of maximum energy efficiency

$$
\begin{aligned}
& \left(k_{1}=1.1 \mathrm{Nm}^{-1}, \tilde{k}_{2}=20 \mathrm{Nm}^{-1}, \text { and } d=1.2 \mathrm{~m}\right) \\
& \rightarrow\left(\bar{v}=0.65 \mathrm{~ms}^{-1}, 1 / \mathrm{COT}=69.75\right) .
\end{aligned}
$$

To have a fair comparison between these cases, the velocity and COT are evaluated in percentage to the nominal case; $3 \mathrm{~kg}$ for the hip and the shoulder. Fig. 6(b) shows that the piecewise linear and the stiff spines $\left(k_{1}=54 \mathrm{Nm}^{-1}\right)$ have better robustness against changes in the masses around the nominal point ( $3 \mathrm{~kg}$ ); compared to the soft linear spine $\left(k_{1}=2.2 \mathrm{Nm}^{-1}\right)$. This means that, the piecewise linear spine designed for the nominal mass is robust against the reasonable changes in the robot's load. This advantageous property of the piecewise linear spine, along with being energy efficient as well as providing fast locomotion (see Fig. 6(a)), is very important in practice.

\section{Behavioral analogy}

The goal of this section is to check if a behavior analogy can be drawn between our locomotion system and a running cheetah [34,35]. Fig. 7(a) shows two most important running states of cheetah and our simple locomotion system. In this figure, as the hip and the shoulder get close to each other, the spine bends upward and the fore and the rear legs are very close to each other. The opposite state happens when the hip and the shoulder, as well as the fore and rear legs, have the maximum distance. Therefore, for the most of the running states, the relative velocity of the upper bodies (shoulder and hip) and the relative velocity of the legs have the same sign. Fig. 7(b) shows the relative velocity of legs versus the relative velocity of upper bodies for different linear spine constants. For $k=1 \mathrm{Nm}^{-1}$ our system has the opposite behavior of a running cheetah. Fig. 3(c) shows that this spring constant results in low energy efficiency and slow locomotion. $k=2.2 \mathrm{Nm}^{-1}$ which results in the fastest locomotion, has the similar behavior to a running cheetah and the same is true for the nonlinear spine. By making the spine stiff, $k=54 \mathrm{Nm}^{-1}$, small upper body movement is attained. So by choosing spring constant for fast locomotion, our locomotion model becomes more like a running cheetah.

The generated spinal motion in our system is sinusoidal-like; specified by frequency, amplitude, and phase lag. It can be seen that the spring constant $\left(k_{1}\right)$ has an interesting effect on the spine phase lag. By spine phase lag here we mean phase difference of the spine and the front leg trajectories. This phase lag can also be extracted by comparing the relative velocity of upper bodies and the relative velocity of lower bodies; such comparison is illustrated in Fig. 7(b). Due to the nonlinearity and discontinuity in our system, the exact mapping between the spring constant and the spine phase lag does not have a closed form solution. However, the effect of spine phase lag on the velocity can be understood through the stride length. Synchronized motion of the spine and the legs, as in cheetah (Fig. 7(a)), will increase the stride length and therefore increases the velocity. Comparing $k=2.2 \mathrm{Nm}^{-1}$ and $k=1 \mathrm{Nm}^{-1}$ in Fig. 7(b) supports this fact. On the other hand, the effect of the engagement locomotion $(d)$ on the spine motion amplitude is quite intuitive. It is obvious that by adding stiffness at location $d$, the spine motion will be limited to this length. Again, this behavior can be seen for the nonlinear case in Fig. 7(b). Finally, at the steady state, the frequency of spine motion is simply equal to the gait frequency.

\section{Experimental results and further simulations}

In this section, we first check whether the conclusions from Section 4 are valid in experiments. A cheap robot is built based on the simple model presented earlier. We call this realization the "crawling robot". Additionally, in this section, we verify whether simulations on a realistic model of a quadruped robot with flexible spine confirm our previous results.

\subsection{Crawling robot}

In order to check the result of simulations conceptually in the real world, we built a simple and low cost crawling robot that behaves abstractly like the proposed model. Nevertheless, the experimental robot works under several uncertainties such as those in slider profiles, bidirectional friction, sensors, and controller.

The robot consists of two parts, fore and rear part, connected with three parallel springs; see Fig. 8(a). Each part has an actuated slider controlled on a sinusoidal trajectory. The slider is being pushed against the ground to generate forward and backward propulsion. The slider-ground friction coefficient is high/low in the backward/forward direction. Each part has three IR sensors providing a low resolution position feedback for the controller. Four passive and low friction wheels sustain each part.

For constructing the piecewise linear spring, we used three sets of different linear springs; see Fig. 8(b). The first spring set (with total stiffness of $\left.k_{1}\right)$ is always engaged. The second $\left(k_{2}\right) /$ third $\left(k_{3}\right)$ 

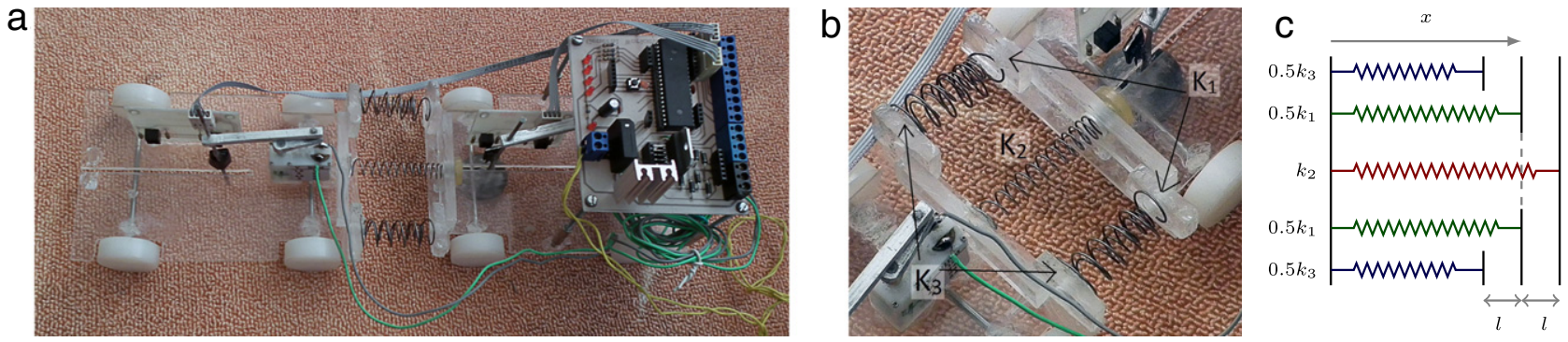

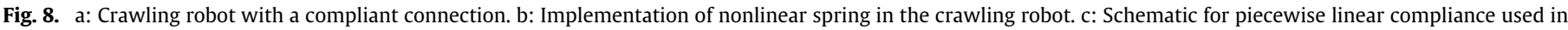
the crawler robot. $l=2 \mathrm{~mm}$.

Table 2

Result for crawling robot.

\begin{tabular}{|c|c|c|c|c|c|c|}
\hline Case & Spring type & $k_{1}(\mathrm{~N} / \mathrm{cm})$ & $k_{2} / k_{1}$ & $k_{3} / k_{1}$ & Crawling time $(\mathrm{s})$ & Performance Improvement over the rigid case \\
\hline 1 & Nonlinear bidirectional, normal & 4.60 & 2.9 & 1.85 & 19.43 & $5.56 \%$ \\
\hline 2 & Nonlinear bidirectional, moderate & 2.64 & 1.64 & 2.48 & 19.88 & $3.17 \%$ \\
\hline 3 & Linear Bidirectional, soft & 2.64 & 0 & 0 & 20.07 & $2.19 \%$ \\
\hline 4 & Nonlinear bidirectional, hard & 7.10 & 2.24 & 1.6 & 20.32 & $0.93 \%$ \\
\hline 5 & Nonlinear bidirectional, soft & 1.42 & 7.18 & 3.8 & 20.48 & $0.15 \%$ \\
\hline 6 & Rigid & $\infty$ & - & - & 20.51 & $0.00 \%$ \\
\hline 7 & Nonlinear in extension & 1.42 & 7.18 & 0 & 20.78 & $-1.30 \%$ \\
\hline 8 & Nonlinear bidirectional, hard & 10.52 & 1.8 & 1.4 & 20.78 & $-1.30 \%$ \\
\hline 9 & Nonlinear bidirectional, hard & 8.61 & 2.0 & 1.5 & 20.83 & $-1.54 \%$ \\
\hline 10 & Nonlinear in compression & 1.42 & 0 & 3.8 & 21.07 & $-2.66 \%$ \\
\hline 11 & linear, very soft & 1.42 & 0 & 0 & 21.16 & $-3.07 \%$ \\
\hline 12 & nonlinear, free in the middle & 0 & $\begin{array}{c}\infty \\
(k 2=8.77)\end{array}$ & $\begin{array}{c}\infty \\
(k 3=3.29)\end{array}$ & 21.57 & $-4.91 \%$ \\
\hline
\end{tabular}

spring set is compressed/extended when the distance of two parts is $2 \mathrm{~mm}$ lower/higher than a nominal distance. Note that third set (with total stiffness of $k_{3}$ ) is located inside the first set; see Fig. 8(b).

In all tests, the source voltage for the controller was the same and the robot was set to crawl a fixed distance. The locomotion time and the electrical current were measured for comparing the effect of different spring sets. Several phase differences between the sliders' positions were tested for different values of the spring constants and among these phase difference, $180^{\circ}$ always led to the best performance. This is consistent with the simulation results. When the phase difference was set to $180^{\circ}$, electrical current remained the same for all of the spring sets $(239 \mathrm{~mA})$ on the power supply. Therefore, the crawling time is used to compare the effect of different spring sets on the energy efficiency and the crawling speed; see Table 2. Note that, the spring set is not exhaustive and the tests were done on the available springs. For each spring, the tests were repeated three times.

In all tests, the nonlinear bidirectional spring sets are better than corresponding linear ones (compare Case 2 with Case 3 and Case 10 with Case 11). In addition, the best case (Case 1 in Table 2) is a nonlinear spring and its performance is $5.56 \%$ better than rigid case. This result is in line with our expectation and the simulation results. Case 12 is the worst one as the two parts are not connected until their relative distance is lower/higher than $2 \mathrm{~mm}$.

Note that friction between the moving parts of each cart is not negligible, the sensory system has a very low resolution, and the motors have low performance. Therefore, the difference between the best and the rigid case is smaller than what was observed in the simulations.

\subsection{Quadruped robot}

\subsubsection{Simulation}

In this set of simulations, energy consumption and speed of a quadruped robot with linear and piecewise linear flexible spines are compared using a more realistic model developed in Webots v6.2. This quadruped is behaviorally related to the simple model presented earlier; both performing bounding gait. The model is similar to the one developed in [17] and Ghostdog model used in [4], but with three serially arranged rotational springs on the spine, see Fig. 9(a). The spring coefficients of the middle and the side springs are denoted by $k_{1}$ and $k_{2}$ respectively. To implement the nonlinear spine characteristic, the rotation of middle spring can be limited to $\left[-\theta_{l},+\theta_{l}\right]$ while the rotations of two other joints are not restricted. It results in nonlinear compliance of spine; see Fig. 9(b). Torque-angle profile of this compliance is complicated, but setting $l$ to zero enables us to calculate this profile as follows and get a feeling of stiffness of such configuration:

$K(\theta)=\left\{\begin{array}{ll}k_{2} / \beta & |\theta| \leq \beta \theta_{l} \\ k_{2} / 2 & \text { elsewhere }\end{array} \quad\right.$ where $\beta=\left(k_{2} / k_{1}+2\right)$.

This profile is illustrated in Fig. 9(c). It can be seen that this profile is similar to the piecewise linear compliance presented earlier. Note that $\beta>2$, so spring always gets stiffer as displacement increases. Despite the difference between translational and rotational springs, from energy point of view, this spine is analogous to the simple model presented earlier in Section 2.

\subsubsection{Results}

The shoulder and the hip joints are active and the rest of the leg joints are passive. The reference trajectories for the shoulder and the hip joints $\left(P_{S}(t)\right.$ and $\left.P_{h}(t)\right)$ are:

$$
\left\{\begin{array}{l}
P_{f}(t)=A \sin (2 \pi f t) \\
P_{h}(t)=A \sin (2 \pi f t+\phi)
\end{array}\right.
$$

where $A=1 \mathrm{rad}$ and $f=1.5 \mathrm{~Hz}$ are the amplitude and the frequency of oscillation and $\phi=-2$ rad is the phase difference. The values of $A, f$ and $b$ are set according to [17]. To compare the nonlinear and the linear spines some sets of simulations for different values of spring coefficients are performed; see Table 3. Energy efficiency improvements are calculated with respect to the rigid case (Case 10). Harder spines cannot be simulated easily due to numerical problems. 

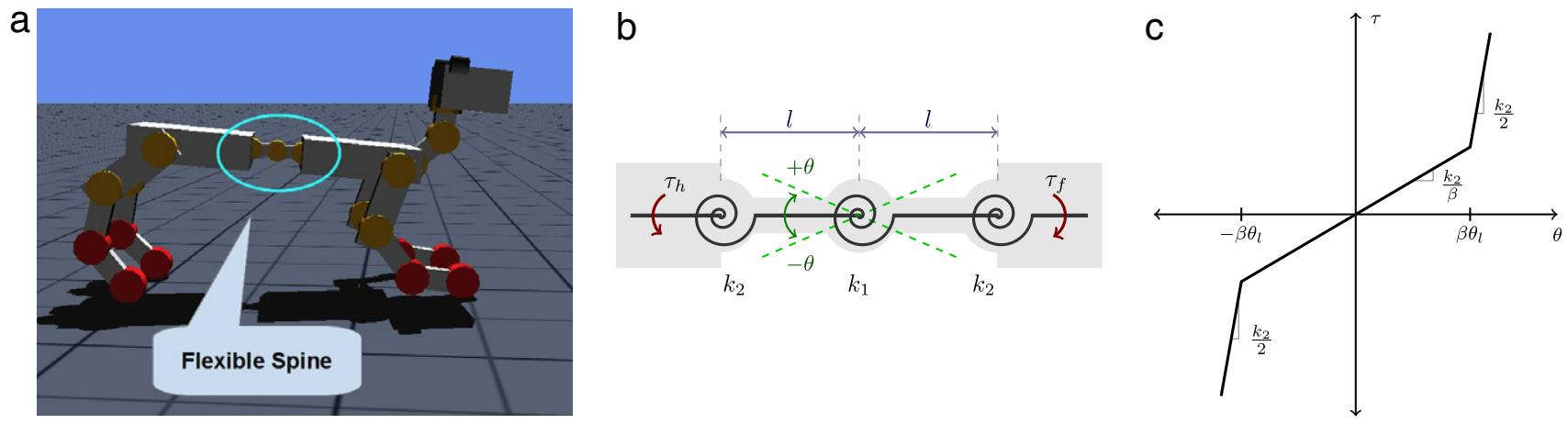

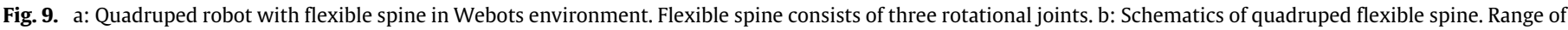
middle joint can be limited. c: Torque-angle profile of quadruped flexible spine when $l=0$. Note that this profile is similar to the one used in Section 4 .

Table 3

Speed and energy efficiency improvement for different spines.

\begin{tabular}{rllcccc}
\hline Case Spring type & $k_{1}$ & $\begin{array}{c}\text { Middle } \\
\text { joint limit }\end{array}$ & $k_{2}$ & $\begin{array}{c}\text { Speed } \\
(\mathrm{m} / \mathrm{s})\end{array}$ & $\begin{array}{c}\text { COT } \\
\text { Improvement } \\
\text { w.r.t. to Case 10 }\end{array}$ \\
\hline 1 & Nonlinear & 100 & $\checkmark$ & $\infty$ & 1.654 & $0.3 \%$ \\
2 & Nonlinear & 100 & $\checkmark$ & 100 & 1.596 & $55.6 \%$ \\
3 & Linear, very soft & 100 & $\times$ & 100 & 1.671 & $2.6 \%$ \\
4 & Linear, soft & $\infty$ & - & 100 & 1.667 & $1.5 \%$ \\
5 & Nonlinear & 100 & $\checkmark$ & 200 & 1.675 & $54.7 \%$ \\
6 & Linear, moderate & 100 & $\times$ & 200 & 1.667 & $1.4 \%$ \\
7 & Linear, moderate & $\infty$ & - & 200 & 1.654 & $0.3 \%$ \\
8 & Nonlinear & 100 & $\checkmark$ & 500 & 1.687 & $53.9 \%$ \\
9 & Linear, moderate & 100 & $\times$ & 500 & 1.665 & $5.7 \%$ \\
10 & Linear, very hard & $\infty$ & - & 500 & 1.645 & $0.0 \%$ \\
\hline
\end{tabular}

In these simulations, the rotation limit of the middle joint $(\theta)$ in the nonlinear spines is set to half value of the spine rotation in Case 7. In each case, the consumed energy at the hip and the shoulder joints and the robot's average speed over 160 cycles of running are logged. Note that, the spinal joints are passive with no energy consumption. As the data in Table 3 show, changes in the spine stiffness do not affect the robot velocity significantly as the hip and the shoulder joint trajectories (the gait) are the same for all settings. Nevertheless, using nonlinear spines have resulted in energy efficiency over the hard spine case (Case 10) up to 55.6\%; see Cases 2, 5 and 8. Common characteristic of these cases is imposing joint limit on the middle joint; that results in nonlinearity in the spine compliance. Note that the variation in the stiffness of side springs - in the nominal range - does not significantly change the energy efficiency. Nevertheless, the results show that making the side joints too stiff results in reduction in the energy efficiency; see Case 1 . These results once again confirm the superiority of piecewise linear spines over the linear ones. Please note that the simple and Webots models are the same just behaviorally, not structurally. Therefore, the results are not comparable numerically. Nevertheless, both models show that nonlinear springs act much better.

\section{Discussions}

\section{Modeling and methodology}

The logic behind our step-by-step approach was to start with a simple, but behaviorally analogous to real systems, model. This simplicity enables us to easily test and analyze our linear and nonlinear spine in simulations and experiments. More realistic models, such as those presented in [36,37], are intractable for a detailed study of spine compliance. Having studied spine compliance on the simple mode, we tested the validity of our results by experimenting on the crawler robot. Although there were differences in dimensions, the simple model and the crawler robot exhibited concurring results. From there, we established our hypotheses and we tested that in Webots simulator on a quadruped robot. Results of that simulation justified our hypothesis that the energy efficiency can be improved using nonlinear spine compliance. Moreover, we are in the process of experimenting our ideas on a real robot; see [20,21]. Also check [38] for more discussions on the nonlinear compliance in robotic applications.

Why nonlinear spine results in velocity/energy efficiency

The results showed that piecewise linear spine has a tremendous effect on the energy efficiency. Among the nonlinear spine parameters, the engagement location $(d)$ plays a key role. This observation can be explained through a simple example; an unforced mass-spring system with an initial mass velocity (Fig. 10(a)). In this system, the kinetic energy $\left(U_{k}\right)$ comes from the mass velocity and the potential energy $\left(U_{p}\right)$ comes from the spring compression. The system starts with $U_{k}=0.5 \mathrm{~J}$ and $U_{p}=0 \mathrm{~J}$. The spring starts compressing from its rest length to the point where all the kinetic energy is transferred to the potential energy $(\dot{x}=0)$. After this point, the process reverses. Fig. 10(b) shows how the compliance affects force-time behavior of the system. The maximum energy stored in the spring is independent of the compliance profile (see Fig. 10(c)), however it is not the case for the profile of kinetic-potential energy transformation and subsequently the spring force profile (see Fig. 10(b)). It means, the compliance profile highly affects the system's motion. In the nonlinear case, most of the energy transferred in the last stage of compression. The same behavior can be found in the force profiles; see Fig. 10(b). The maximum forces $\left(F_{\max }\right)$ and the period of motion $(T)$ are the interesting points in this figure. In the linear case, the maximum force (the period of motion) is directly (inversely) proportional to the spring constant. However, in the nonlinear case, these two quantities can be controlled independently. Moreover, in the piecewise linear case, the ratio of energy transformation before and after engagement of the stiff part $(d)$ can be controlled too. It means that improving the energy efficiency and increasing the velocity are decoupled. That is, nonlinear spine results in velocity/energy efficiency.

\section{Conclusions}

In this paper, we presented a simple locomotion system which is behaviorally and structurally similar to a galloping quadruped. Using this model, we showed that fast locomotion requires flexible spine. We, thereafter, demonstrated that both fast locomotion and energy efficiency cannot be attained with the same linear spines. Nevertheless, this goal can be partially achieved when a nonlinear spine is used. In other words, nonlinear spine results in good tradeoff between energy efficiency and fast locomotion. Our model 

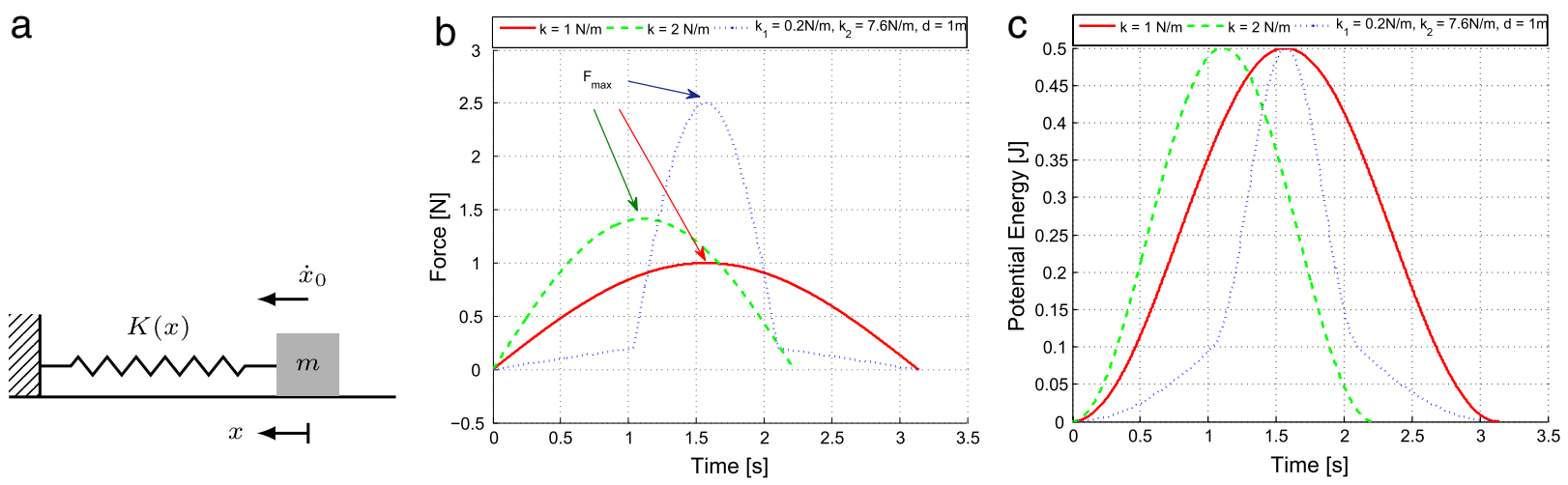

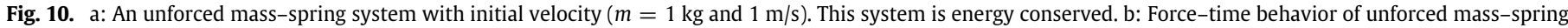
system for different springs. b: Potential energy stored in spring over time.

of nonlinear spine is as simple as possible; a flexible linear spring which is limited by two stiff springs. The intuition behind this structure is compliance behavior of muscle-tendon. We tested our idea of nonlinear flexible spine for a quadruped in Webots as well. As the results showed, the energy efficiency was improved while the average velocity was almost constant. This was mostly in line with the behavior of our simple locomotion system.

Based on unreported extensive simulations, the discussed superiority of the piecewise linear spines over the linear ones is gait independent. Nevertheless, the optimal values of spring constants and the engagement location vary by gait. Therefore, the next stage of this research is to synthesize an adaptive flexible spine to attain high energy efficiency and fast locomotion at different gaits. Moreover, the methodology used in the paper is experimental and simulation oriented. Using a theoretical approach to the optimal design of spine compliance is on the list of our future researches.

\section{Acknowledgment}

Authors would like to thank University of Tehran for supporting this research.

\section{References}

[1] T. McGeer, Passive dynamic walking, International Journal of Robotics Research 9 (1990) 62-82.

[2] J. Buchli, A.J. Ijspeert, Self-organized adaptive legged locomotion in a compliant quadruped robot, Autonomous Robots 25 (2008) 331-347.

[3] F. Iida, R. Pfeifer, Self-stabilization and behavioral diversity of embodied adaptive locomotion, in: Embodied Artificial Intelligence, Springer, 2004, pp. 119-129.

[4] L. Righetti, A.J. Ijspeert, Pattern generators with sensory feedback for the control of quadruped locomotion, in: Proceedings of International Conference on Robotics and Automation 2008, ICRA, 2008, pp. 819-824.

[5] A. Schmitz, G. Gmez, F. Iida, R. Pfeifer, On the robustness of simple speed control for a quadruped robot, in: Proceedings of International Conference on Morphological Computation 2007, 2007, pp. 88-90.

[6] J. Buchli, F. Iida, A.J. Ijspeert, Finding resonance: Adaptive frequency oscillators for dynamic legged locomotion, in: Proceedings of International Conference on Intelligent Robots and Systems 2006, IROS, 2006, pp. 3903-3909.

[7] I. Poulakakis, J.W. Grizzle, The spring loaded inverted pendulum as the hybrid zero dynamics of an asymmetric hopper, Transactions on Automatic Control 54 (2009) 1779-1793.

[8] B. Vanderborght, R. Van Ham, B. Verrelst, M. Van Damme, D. Lefeber, Overview of the lucy project: dynamic stabilization of a biped powered by pneumatic artificial muscles, Advanced Robotics 22 (2008) 1027-1051.

[9] B. Vanderborght, R. Van Ham, D. Lefeber, T.G. Sugar, K.W. Hollander, Comparison of mechanical design and energy consumption of adaptable, passive-compliant actuators, International Journal of Robotics Research 28 (2009) 90-103.

[10] B. Vanderborght, Dynamic Stabilisation of The Biped Lucy Powered by Actuators With Controllable Stiffness, Vol. 63, Springer, 2010.

[11] H. Inoue, Flexible spine robot and tendon-driven humanoid, 2003. URL: http://www.jsk.t.u-tokyo.ac.jp/ ikuo/msm/200001/index-e.html.
[12] I. Mizuuchi, R. Tajima, T. Yoshikai, D. Sato, K. Nagashima, M. Inaba, Y. Kuniyoshi, H. Inoue, The design and control of the flexible spine of a fully tendon-driven humanoid, in: Proceeding of International Conference on Intelligent Robots and Systems 2002, IROS, 2002, pp. 2527-2532.

[13] I. Mizuuchi, S. Yoshida, M. Inaba, H. Inoue, The development and control of a flexible-spine for a human-form robot, Advanced Robotics 17 (2003) 179-196.

[14] G. Folkertsma, S. Kim, S. Stramigioli, Parallel stiffness in a bounding quadruped with flexible spine, in: Proceeding of International Conference on Intelligent Robots and Systems 2012, IROS, 2012, pp. 2210-2215.

[15] BostonDynamics, 2013. Bd. URL: http://www.bostondynamics.com/robot cheetah.html.

[16] J.M. Gl, Spinal flexion and locomotor energetics in Kangaroo, Monkey, and Tiger, Canadian Journal of Zoology 70 (1992) 2444-2451.

[17] H.J. Bidgoly, A. Vafaei, A. Sadeghi, M.N. Ahmadabadi, Learning approach to study effect of flexible spine on running behavior of a quadruped robot, in: Proceeding of International Conference on Climbing and Walking Robots 2010 CLAWAR, 2010, pp. 1195-1201.

[18] M.H.H. Kani, M. Derafshian, H.J. Bidgoly, M.N. Ahmadabadi, Effect of flexible spine on stability of a passive quadruped robot: Experimental results, in: Proceeding of International Conference on Robotics and Biomimetics 2011, ROBIO, 2011, pp. 2793-2798.

[19] M.H.H. Kani, M. Nili Ahmadabadi, Comparing effects of rigid, flexible, and actuated series-elastic spines on bounding gait of quadruped robots, in: Proceeding of International Conference on Robotics and Mechatronics 2013, ICRoM, 2013, pp. 282-287.

[20] M. Khoramshahi, A. Sprowitz, A. Tuleu, M.N. Ahmadabadi, A. Ijspeert, Benefits of an active spine supported bounding locomotion with a small compliant quadruped robot, in: Proceedings of International Conference on Robotics and Automation 2013, ICRA, 2013.

[21] A. Sprowitz, E. Badri, M. Khoramshahi, A. Tuleu, A. Ijspeert, Use your spine! Effect of active spine movements on horizontal impulse and cost of transport in a bounding, quadruped robot, in: Proceedings of Dynamic Walking 2013 2013.

[22] A.A. Biewener, Tendons and ligaments: structure, mechanical behavior and biological function, in: Collagen, Springer, 2008, pp. 269-284

[23] J. Buchli, A.J. Ijspeert, A simple, adaptive locomotion toy-system, From Animals to Animats 8 (2004) 153-162.

[24] K.F. Leeser, Locomotion experiments on a planar quadruped robot with articulated spine, Ph.D. Thesis, Massachusetts Institute of Technology, 1996.

[25] A.J. Ijspeert, Central pattern generators for locomotion control in animals and robots: a review, Neural Networks 21 (2008) 642-653.

[26] A.J. Ijspeert, A connectionist central pattern generator for the aquatic and terrestrial gaits of a simulated salamander, Biological Cybernetics 84 (2001) 331-348.

[27] C.R. Taylor, K. Schmidt-Nielsen, J.L. Raab, Scaling of energetic cost of running to body size in mammals, American Journal of Physiology-Legacy Content 219 (1970) 1104-1107.

[28] C.R. Taylor, A. Shkolnik, R. Dmi'el, D. Baharav, A. Borut, Running in Cheetahs, Gazelles, and Goats: energy cost and limb configuration, American Journal of Physiology-Legacy Content 227 (1974) 848-850.

[29] K. Schmidt-Nielsen, Scaling: Why is Animal Size so Important? Cambridge University Press, 1984.

[30] P. DeVita, J. Helseth, T. Hortobagyi, Muscles do more positive than negative work in human locomotion, Journal of Experimental Biology 210 (2007) 3361-3373.

[31] D.F. Hoyt, C.R. Taylor, Gait and the energetics of locomotion in horses, Nature 292 (1981) 239-240.

[32] A. Schepelmann, H. Geyer, A synthesis method for compact nonlinear springs with custom torque-deflection profiles and bandwidth for series elastic actuators, in: Proceedings of Dynamic Walking 2013, 2013.

[33] S.P. Boyd, L. Vandenberghe, Convex Optimization, Cambridge University Press, 2004.

[34] M. Hildebrand, Motions of the running Cheetah and Horse, Journal of Mammalogy 40 (1959) 481-495.

[35] M. Hildebrand, Further studies on locomotion of the Cheetah, Journal of Mammalogy (1961) 84-91. 
[36] Z. Gan, C.D. Remy, A simplistic model for quadrupedal walking and trotting, in: Proceedings of Dynamic Walking 2013, 2013.

[37] C.D. Remy, Optimal exploitation of natural dynamics in legged locomotion, Ph.D. Thesis, ETH Zurich, 2011.

[38] M. Khoramshahi, A study on effects of flexible spine on stability and energy consumption of a quadruped robot, Master's Thesis, University of Tehran, Iran, 2012.

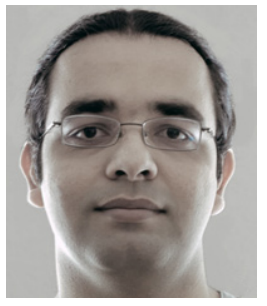

Mahdi Khoramshahi received his B.Sc. degree in the Electrical Engineering from Sharif University of Technology (2004-2009). He received his M.Sc. in Electrical Engineering from University of Tehran (2009-2012). He carried out as an intern in Biorobotics Laboratory (BioRob) at EPFL under Prof. Auke Ijspeert supervision (2011-2012). He then became a research scholar at NanoRobotics laboratory under Prof. Metin Sitti supervision at Carnegie Melon University (2012-2013). His research interests are robotics, dynamics systems and control, nonlinear systems and machine learning.

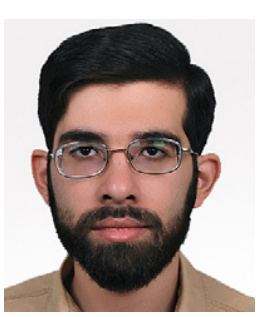

Hamed Jalaly Bidgoly received his B.Sc. in Electrical Engineering, Control field and his M.Sc. in Intelligence Control from University of Tehran, Iran, in 2009 and 2011 , respectively. He is currently a Ph.D. student of Robotics and Machine Intelligence at University of Tehran. His research interests are nonlinear control in robotics and legged locomotion.

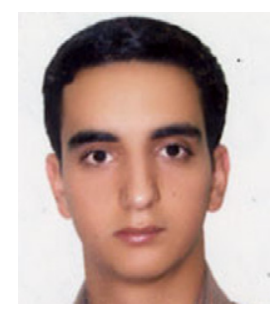

Soroosh Shafiee was born in September 1989 in Abadeh, Iran. He received his B.S. degree in Electrical Engineering from University of Tehran in May 2011. He is currently a graduate student in Electrical Engineering at the Schoo of ECE, University of Tehran, Tehran, Iran. His research interests are Pattern Recognition, machine learning, time series forecasting and Robotics.

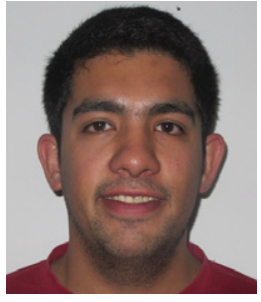

Ali Asaei received his B.Sc degree in the Electrical Engineering from the University of Tehran (2007-2012). Now he is working on the pole climber robot project under the supervision of Dr. Nili in the RAIIS (Robotics, Artificial Intelligent and Information Science) lab. His research interests include the mobile robots, climbing robots, manipulators, and motion planning. He was a member of the UT Solar Car team (2008-2011) which participated in WSC (World Solar Challenge) 2011, in Australia.

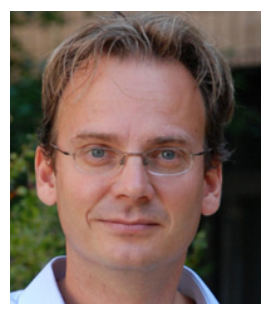

Auke Jan Ijspeert is an associate professor at the EPFL (the Swiss Federal Institute of Technology at Lausanne), and Head of the Biorobotics Laboratory (BioRob), previously the Biologically Inspired Robotics Group (BIRG). He has a B.Sc/M.Sc in Physics from the EPFL, and a PhD in artificial intelligence from the University of Edinburgh. He carried out postdocs at IDSIA and EPFL (LAMI) with Jean-Daniel Nicoud and Luca Gambardella, and at the University of Southern California (USC), with Michael Arbib and Stefan Schaal. He then became a research assistant professor at USC, and an external collaborator at ATR (Advanced Telecommunications Research institute) in Japan. In 2002, he came back to the EPFL first as a SNF assistant professor, and since October 2009 as an associate professor (with tenure). His research interests include the intersection between robotics, computational neuroscience, nonlinear dynamical systems, and applied machine learning.

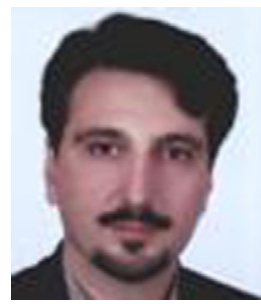

Majid Nili Ahmadabadi was born in 1967 and received his B.S in Mechanical Engineering from Sharif University of Technology of Iran in 1990. He received his M.Sc. and Ph.D. in Information Sciences from the Graduate School of Information Science, Tohoku University, Japan in 1994 and 1997 respectively. In 1997, he joined the Advanced Robotics Laboratory at Tohoku University. Later he moved to the Department of Electrical and Computer Engineering Faculty of Engineering, University of Tehran where he is a professor and the Head of Robotics and AI and Cognitive Robotics Laboratories. He is also a senior researcher at the School of Cognitive Sciences, Institute for studies on Theoretical Physics and Mathematics, Tehran, Iran. His research interest include learning in multi-agent systems, cognitive robotics, biologically inspired learning methods, distributed robotics, object manipulation, and mobile robots. 